\title{
Reliability for Drug Targeting In Cancer Treatment through Nanotechnology (A Psychometric Approach)
}

\author{
Nader Ebrahimi' and Mansoori, G Ali \\ ${ }^{1}$ Division of Statistics, Northern Illinois University, USA \\ ${ }^{2}$ Departments of Bioengineering, Chemical Engineering and Physics, University of Illinois at Chicago, USA
}

*Corresponding author: Mansoori, G Ali, Departments of Bioengineering, Chemical Engineering and Physics, University of Illinois at Chicago, Illinois 60607, USA, Tel: 312-996-5592; E-mail: mansoori@uic.edu

\begin{abstract}
The primary focus of the nanotechnology therapeutic approaches has been on early disease detection, drug discovery and monitoring, controlled release of therapeutic agents, and targeted drug delivery. Targeted drug delivery system requires more research including statistical modeling to establish the reliability in success of drug targeting. This part of every nanotechnology therapeutic approach is especially fundamental for reaching stronger therapeutic effects with lower side effects.

In practice, we may be unable to accurately assess our nanotechnology drug delivery system designs to improve the reliability. This is likely due to the lack of a unified definition of reliability in system design phase. For this shortcoming, we introduce the notion of reliability in this context. Our proposed notion of reliability is flexible in allowing a general method for determining dose level. We then describe a methodology that enables us to determine an optimal dose level to achieve certain reliability. Although, in this report we focus on a special nanotechnology drug delivery system for cancer therapy, i.e. folate-conjugated gold nanoparticles for transfer to over expressed-folate-receptor cancerous cells, our developed strategies can be applied to many other drug delivery systems as well.
\end{abstract}

\section{Keywords}

Poisson distribution; Binomial distribution; Nanoconjugate; Folate receptor.

\section{Introduction}

It goes without saying that advances in life- and physical-sciences and engineering have contributed greatly to the improvements made in our quality of life and wellbeing. Molecular-based study of processes is the basis of modern life and physical-sciences and engineering. In the 20th century, technological approaches to solve problems of healthcare, quality of life and human communications started its alteration from a merely rational science to a scientific technology with fully atomic and molecular basis. At first, antibiotics which meddled with pathogens at the molecular level were engineered and introduced. Then, the ongoing developments in chemical and statistical physics, genomics, and now nanobiotechnology have been providing ever more precise knowledge of the functions of the

\begin{tabular}{|c|c|}
\hline$c$ & $\begin{array}{l}\text { the number of nanoconjugates that are carrying the drug and } \\
\text { they are injected into the blood stream }\end{array}$ \\
\hline$p^{*}$ & $\begin{array}{l}\text { the probability that any of } c \text { nanoconjugates be able to } \\
\text { penetrate the tissue }\end{array}$ \\
\hline$\lambda_{1}$ & $\begin{array}{l}\text { the average number of folate receptors for a healthy cell in } \\
\text { the tissue }\end{array}$ \\
\hline$\lambda_{2}$ & $\begin{array}{l}\text { the average number of folate receptors for a cancerous cell } \\
\text { in the tissue }\end{array}$ \\
\hline$\lambda$ & the average number of cells in the tissue \\
\hline$p$ & the chance of a cell being a cancerous cell in the tissue \\
\hline$\frac{p^{*} \lambda_{2}}{\left(\lambda_{1}+\lambda_{2}\right)}$ & $\begin{array}{l}\text { the chance of a nanocojugate that is carrying the drug to } \\
\text { penetrate a cancerous cell in the tissue }\end{array}$ \\
\hline $\mathrm{k}$ & $\begin{array}{l}\text { the number of nanocojugates, carrying the drug, needed to } \\
\text { destroy a cancerous cell in the tissue }\end{array}$ \\
\hline$\Phi$ & $\begin{array}{l}\text { the cumulative distribution function of the standard normal } \\
\text { distribution }\end{array}$ \\
\hline $\mathrm{z}_{\alpha}$ & the $\alpha$ percentile of the standard normal distribution. \\
\hline
\end{tabular}

different micro and macro organism, and mechanisms underlying living things, infections and diseases at the atomic and molecular level. In other words, understanding of life advanced from understanding the functions of organs and tissues to the functions of cells and finally nano scale systems as well as supra-, macro- and individualmolecules [1-8].

Nanotechnology developments were initiated during the last decade of the 20th century when we had already achieved profound molecular awareness about the living systems in general and human body in particular. Generally, nanotechnology is the science of dealing with molecular scale systems and matters. Such systems and matters being worked on through nanotechnology have the following three important features [2].

(a) They have at least one of their three dimensions in between 1 to $200 \mathrm{~nm}$.

(b) There are some techniques with control on the physical and chemical characteristics of structures in molecular scale.

(c) They are able to be assembled together to generate larger

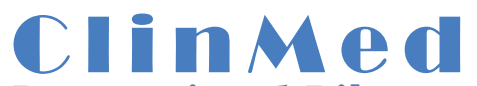

International Library

Citation: Ebrahimi N, Mansoori GA (2014) Reliability for Drug Targeting In Cancer Treatment through Nanotechnology (A Psychometric Approach). Int J Med Nano Res 1:004

Received: October 03, 2014: Accepted: November 25, 2014: Published: November 28, 2014 


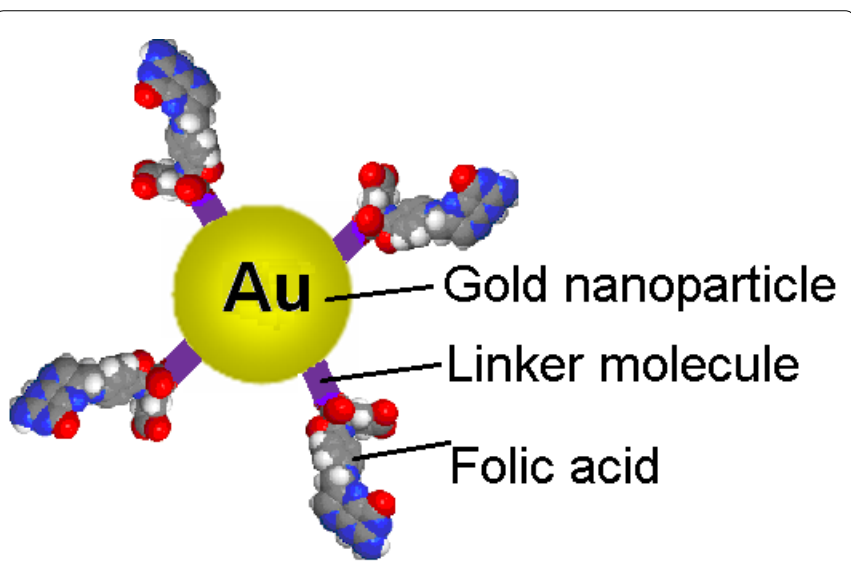

Figure 1: Nanoconjugate formed by folic acids or folates, attached to gold nanoparticles through a linker like a thiol molecule [9].

structures.

These features are applicable to all natural and microbiological systems including bio-systems such as cells, bacteria, enzymes and viruses.

The simultaneity between the launch of nanotechnology development and our molecular awareness of living systems has resulted in introducing a molecular-based technological medicine in which the molecular basis of life is manipulated to construct specific desired results through nanotechnology methods and devices. In other words, the knowledge of human molecular structure is used to design biomedically active microscopic devices in the $21^{\text {st }}$ century. These devices will be used for missions of cellular inspection, repair, and reconstruction. Therefore, the $21^{\text {st }}$ century treatment through nanobiotechnology is expected to entail the proliferation of efficacious therapeutic molecular tools to establish and maintain a continuous state-of-health for humans $[1,3]$.

Accumulating evidence suggests that lack of balance between proliferation and apoptosis may cause cancer emergence [4]. Suppression of apoptosis hinders many forms of cancer therapeutic strategies, including radiation therapy, cancer immunotherapy and chemotherapy [5]. Elimination of cancer cells by early apoptosis is preferred over other forms of cell growth inhibition. Apoptosis directly leads to tumor regression and reduces risks of selecting more aggressive and/or drug resistant phenotypes (any observed quality of an organism) that are often responsible for tumor re-growth and treatment failure [6]. Apoptosis also plays a critical role in the cytotoxic activity of a wide range of anticancer agents, including chemotherapeutic agents, biological agents and hormones. Defects in apoptosis pathways are associated with drug resistance in many cancers [7].

Generally, the focus of the nanotechnology therapeutic approaches to treat cancer has been on early disease detection, drug discovery and monitoring, controlled release of therapeutic agents, and targeted drug delivery. Targeted drug delivery requires more research and it is especially fundamental for reaching stronger therapeutic effects with lower side effects. This part of every nanotechnology therapeutic approach requires statistical modeling to establish the reliability in success of drug targeting. In this paper we first introduce the notion of reliability for a nanotechnology drug delivery system. We then report on our efforts to determine an optimal dose of the drug to achieve a particular reliability for a cancer nanotechnology drug delivery system.

This paper is organized as follows. In Section 2 for a specific number of nanoconjugates that are carrying the drug and they are injected into the blood stream, we present a dynamic model for the number of nanoconjugates that penetrated cancerous cells population in the tissue. Similar to our proposed model was used by [8] to model the number of nanoconjugates that might attach to surface of a cell.
In section 3, we introduce the notion of reliability and give algorithm to compute an optimum dose level to achieve certain reliability. Several examples are given in this section to illustrate our proposed methodology. Concluding remarks are provided in Section 4.

\section{Dynamic Model for the Number of Nanoconjugates Penetrated Cancerous Cells Population in a Tissue}

Let us consider a nanoconjugate containing the drug, (for example folic acid connected to a cancerous cell killing agent or gold nanoparticle which the hypothermia agent, or drug, through a linker, like a thiol molecule (Figure 1)), which has potential to enter a cancerous cell in a tissue that has on its surface over-expressed folate receptors $[9,10]$.

Suppose $c$ is the number of these nanoconjugates that are injected into the blood stream. Now, out of $c$ nanoconjugates, let $L$ be a discrete random variable representing the number of nanoconjugates that permeate a tissue. Then, $L$ has Binomial distribution with the probability distribution function.

$$
P(L=l)=\frac{c !}{(l !)(c-l) !}\left[p^{*}\right]^{c-l}\left[1-p^{*}\right]^{c-l}, l=0,1,2,3, \ldots . . \infty
$$

where $p^{*}$ is the probability of any nanoconjugte carrying the drug being able to penetrate the tissue. From the equation (2.1), the average number of nanoconjugates that reach the tissue is $c p^{*}$ [11]. It is worth mentioning that in practice, $p^{*}$ is usually a function. of velocity of a blood flow. Velocity is a function of $(x, y, z)$, but when it gets to cell it really does not matter if it has vectorial character, but for the macroscopic system it is a vector.

Let $X$ and $Y$ be the number of folate receptors for healthy and cancerous cells, respectively. Since cancerous cells are normally bigger than normal cells, i.e., a cancerous cell has more folate receptors than an healthy cell, so that $X$ is stochastically smaller than $Y$ [12]. To study stochastic behaviors of both random quantities, we assume that $X$ and $Y$ have Poisson distributions with parameters $\lambda_{1}$ and $\lambda_{2}$, respectively. Here, the parameter $\lambda_{1}$ is the average number of folate receptors for a healthy cell and the parameter $\lambda_{2}$ is the average number of folate receptors for a cancerous cell and $\lambda_{1} \leq \lambda_{2}$. For more details about Poisson distribution see [11]. In reality, cancerous cells and healthy cells are not well distributed in the tissue. That is, the number of healthy cells and cancerous cells are both dynamic. Thus, stochastic models needed to model the number of cancerous cells as well as healthy cells in the tissue. For that, we let the total number of cells in the tissue be represented by $Z$, where $Z$ has Poisson distribution with the parameter $\lambda$. The parameter $\lambda$ is the average number of cells in the tissue. Suppose the chance of a cell being a cancerous cell is $p$ and the chance of being an healthy cell is $1-p$. Then, $Z_{1}$, the size of cancerous cells population in the tissue, is Poisson with parameter $\lambda \mathrm{p}$ and $Z_{2}$, the size of healthy cells population in the tissue, is Poisson with the parameter $\lambda(1-p)$. The parameters $\lambda p$ and $\lambda(1-p)$ represent average numbers of cancerous cells and healthy cells in the tissue, respectively.

Define a binary random variable $W$ such that $W=1$ if a nanoconjugate which is already inside the tissue enters a cancerous cell and $\mathrm{W}=0$ if it enters a healthy cell. Then, $P(W=1)$ is approximately equal to $\frac{\lambda_{2}}{\left(\lambda_{1}+\lambda_{2}\right)}$ (Appendix 1). Since a cancer nanotechnoloy drug delivery system is applicable when $\lambda_{1}$ is much smaller than $\lambda_{2}$, i.e., $\lambda_{1}<<\lambda_{2}$, therefore the chance of a nanoconjugate penetrates a cancerous cell is much higher than an healthy cell. Suppose $L_{l}$ represents the number of nanoconjugates that reach the cancerous cells population in the tissue, then one can proof that $L_{1}$ has Binomial distribution with parameters $c$ and $\frac{p^{*} \lambda_{2}}{\left(\lambda_{1}+\lambda_{2}\right)}$. That is, the average number of nanoconjugates that reach the cancerous cells population in the tissue is $c \times \frac{p^{*} \lambda_{2}}{\left(\lambda_{1}+\lambda_{2}\right)}$ (Appendix 2). It should be noted that this number is expected to be much higher than the average number of nanoconjugates, $c \times \frac{p^{*} \lambda_{1}}{\left(\lambda_{1}+\lambda_{2}\right)}$ that reach healthy cells population in the 
Table 1: Values of $B(K, C)$ for given values of $C$ and $k$.

\begin{tabular}{|c|c|c|}
\hline$k$ & $c$ & $B(k, c)$ \\
\hline 1 & 1 & 0.8182 \\
\hline 1 & 2 & 0.9669 \\
\hline 1 & 3 & 0.9939 \\
\hline 1 & 4 & 0.9989 \\
\hline 1 & 5 & 0.9998 \\
\hline 1 & more than 5 & 1 \\
\hline 3 & 3 & 0.5485 \\
\hline 3 & 4 & 0.8465 \\
\hline 3 & 5 & 0.9550 \\
\hline 3 & 6 & 0.9880 \\
\hline 3 & 7 & 0.9970 \\
\hline 3 & 9 & 0.9993 \\
\hline 3 & 10 & 0.9998 \\
\hline 3 & more than 10 & 0.9999 \\
\hline 3 & & 1 \\
\hline
\end{tabular}

tissue.

Since the particular mechanism can make a big difference in the number of nanoconjugates delivered to the cancerous cells and since different modes of photo dynamic therapy require a different number of nanoconjugates needed to kill a cancerous cell, we assume that one needs $\mathrm{k}$ nanoconjugates to penetrate a cancerous cell and kill it $[13,14]$. Here $\mathrm{k}$ is provided by an expert. Now, the chance of a cancerous cells population having size $1, Z_{1}=1$, being killed for given $c$ is

$$
B(k, c)=\left[\sum_{i=k}^{c} b(i, c)\right],
$$

where

$$
b(i, c)=\frac{c !}{(i !(c-i) !)}\left[\left(\frac{p^{*} \lambda_{2}}{\lambda_{1}+\lambda_{2}}\right)^{i}\left(1-\frac{p^{*} \lambda_{2}}{\lambda_{1}+\lambda_{2}}\right)^{c-i}\right]
$$

Table 1 gives values of $B(k, c)$ for different values of $k$ and $c$ when $\lambda_{2}=10, \lambda_{1}=1$ and $p^{\star}=0.9$. Binomial Table can be used for other values of parameters.

For example from Table 1 , given that we need $k=3$ nanoconjugates to kill a cancerous cell, six nanoconjugates, $c=6$ will kill the cancerous cell with probability 0.988 . That is, six nanoconjugates should be injected. As discussed [14], in some situations the size of a nanoconjugate is much smaller than the size of a cancerous cell and therefore one needs to deliver hundreds of nanoconjugates to kill a cancerous cell. So in (2.2) if we take $k=500$, then we need to inject $c=743$ nanoconjugates to make sure that the cancerous cell will be destroyed with probability 0.988 . Again we emphasize here that it is assumed the tissue has only one cancerous cell and about $82 \%$ of injected nanoconjugates penetrated the cancerous cell.

\section{Notion of Reliability and Criteria to Find C}

Let $N$ be the number of cancerous cells in the tissue that will be killed with $c$ injected nanoconjugates. Then we call a nanotechnology drug delivery system to be $100 \alpha$ percent reliable if $P\left(N=Z_{1}\right)=\alpha$. Statistically speaking, the system's reliability is $\alpha$ if we are $100 \alpha \%$ confident that all cancerous cells in the tissue will be destroyed by this nanotechnology drug delivery system. It is worth mentioning that our definition of a reliability is different from the classical definition of reliability where a system's reliability is defined in-terms of its lifetime [15]. Our proposed criteria to find an optimal $\mathrm{c}$ is: Find the smallest $\mathrm{c}$ that achieves $100 \alpha \%$ Reliability.

Using our criteria, $\mathrm{c}$ must satisfy the following equation.

$$
P\left(N=Z_{1}\right)=\sum_{z=0}^{\infty} P\left(N=z \mid Z_{1}=z\right) \frac{\exp (-\lambda p)(\lambda p)^{z}}{z !}=a .
$$

Now in the equation (3.1), we need to calculate $P\left(N=z \mid Z_{1}=z\right)$ Using the equation (2.2), it is clear that $P\left(N=0 \mid Z_{1}=0\right)=1$ and $P$ $\left(N=1 \mid Z_{1}=1\right)=B(k, c)$. Given that a cancerous cell is killed, suppose the chance that it was killed by $j$ nanoconjugates is, $\mathrm{a}_{\mathrm{j}}$ where $j=k, k$ $+1, \ldots$, that is, $P$ (a cancerous cell was killed by $j$ nanoconjugates|a cancerous cell was killed) $=\mathrm{a}_{\text {. }}$ Throughout this paper we assume that $a_{k}=1$. That is, given a cancerous cell is killed, the chance that it was killed by $\mathrm{k}$ nanoconjugates is $100 \%$. Since $c$ is usually large, using Poisson approximation for Binomial distribution, we can assume that $L_{1}$, the number of nanoconjugates that reach cancerous cells population in the tissue, is Poisson with the mean

$$
A^{*}=c \times \frac{p^{*} \lambda_{2}}{\left(\lambda_{1}+\lambda_{2}\right)} .
$$

Thus,

$P\left(N=z \mid Z_{1}=z\right)=P\left(L_{1} \geq k z \mid Z_{1}=z\right)=\sum_{j=k z}^{\infty} \frac{\exp \left(-A^{*}\right)\left(A^{*}\right)^{j}}{j !} \approx\left(1-\Phi\left(\frac{k z-A^{*}}{\left(A^{*}\right)^{-5}}\right)\right)_{3.3}$

In the equation (3.3), $\Phi$ stands for the cumulative distribution function of the standard normal distribution function [12].

In the appendix 3 , we provide $\mathrm{R}$-codes to calculate the middle part of the equation (3.1) using the equation (3.3) [16]. Applying this program, one can calculate the reliability of a nanotechnology drug delivery system. The following example illustrates that.

Example 1: Suppose we have designed a nanotechnology drug delivery system with $c=420, k=3, \lambda=1000, p=0.1, \lambda_{2}=20, \lambda_{1}=1$ and $p^{*}=0.95$. Using the R-program we get $P\left(N=Z_{1}\right)=0.98$. That is, this system is $98 \%$ reliable. However, if we move from $p=0.1$ to $p=0.9$, then the system is $0 \%$ reliable. That is this drug delivery system is highly unreliable.

Now to obtain our optimal $c$ that achieves $100 \alpha \%$ confidence, we propose the following algorithm.

\section{Algorithm}

(a): Choose an initial value for $c$ say $c=c_{0}$, then compute $p\left(N=Z_{1}\right)$ using our R-program. If this probability is less than $\alpha$, take $c=c_{0}+1$ and compute the probability again. Continue this until for the first time the probability is greater than or equal to $\alpha$.

(b): If for $c_{0}$, the probability is more than $\alpha$. Then, take $c=c_{0}-1$ and compute the probability again. Continue this until for the first time the probability is less than or equal to $\alpha$. Suppose $c^{\star}$ is the answer. Now, if for $c=c^{\star}$, the probability is exactly $\alpha$, then the optimal solution is $c^{\star}$. If it is less than $\alpha$, then the optimal solution is $c=c^{\star}+1$

Several comments are in order with regard to our proposed Algorithm.

(1) To implement the algorithm, we need to provide $k, \lambda, p, \lambda_{2}$, $\lambda_{1}$ and $p^{*}$.

(2) Our recommendation is as an initial value for $c$ take $c_{0}=(k$ $\lambda p)+\left(\mathrm{z}_{\alpha} \mathrm{k}(\lambda \mathrm{p})^{0.5}\right)$, where $\mathrm{za}$ is a percentile of the standard normal distribution.

(3) To calculate probabilities for Binomial and Poisson distributions sometimes one needs to use Normal approximations for both distributions [11].

The following example illustrates implementation of our Algorithm.

Example 2: In this example, we describe four different scenarios.

(a) Let $k=3, \lambda=1000, p=0.1, \lambda_{2}=20, \lambda_{1}=1$ and $p^{*}=0.95$. Suppose our goal is to design a nanotechnology drug delivery system that achieves $95 \%$ reliability. To find $c$ that guarantees such reliability, as mentioned in (2), we start with $c_{0}=360$. Using our R-program, for this number the reliability is $75.5 \%$. So to increase the reliability, we move to $c_{0}=361$. Still for $c_{0}=361$ the reliability is lower than $95 \%$. So we 
continue until we reach the threshold $95 \%$. For this nanotechnology drug delivery system, the optimal solution is $c=400$. That is, one needs at least 400 nanoconjugates to inject into the blood stream to achieve the reliability of $95 \%$.

(b) In the part (a), take $p=0.90$ and keep the values of other parameters the same. That is, we assume that $90 \%$ of cells in the tissue are cancerous cells. Applying our Algorithm, we start with $c_{0}=2880$ for, 2880 nanoconjugates the reliability is $15 \%$ which is much lower than $95 \%$. We continue increasing number of nanoconjugates until we get $95 \%$. The optimal solution is $c=3191$. That is, we need 3191 nanoconjugates to inject into blood stream to achieve the reliability of $95 \%$.

(c) In the part (a), take $p=0.90$ and $k=1$ and keep the values of other parameters the same. That is, assume that $90 \%$ of cells in the tissue are cancerous cells and to kill a cancerous cell one nanoconjugate is required. Applying our algorithm, we start with $c_{0}=960$. For, 960 nanoconjugates the reliability is $20 \%$ which is smaller than $95 \%$. We increase the number of nanoconjugates in order to achieve our goal of $95 \%$ reliability. The optimal answer is 1080 . It is interesting to note that by moving from $k=3$, part (b), to $k=1$, the dose level (the number of nanoconjugates) needed to achieve the same reliability is much less.

(d) In the part (a), take $p=0.9$, and keep the values of other parameters the same. If we want our system to be $99 \%$ reliable, we need 4028 nanoconjugates.

\section{Concluding Remarks}

. In this paper, we have introduced a unified definition of reliability for a nanotechnology drug delivery system. This notion provides a general framework for carrying out determination of unknown dose level in order to achieve a specific reliability for a nanotechnology drug delivery system. Our proposed method is very flexible and easy to use. As one expects our finding shows that, one can achieve a specific reliability with low dose level by having small $k$, the number of nanoconjugates needed to kill a cancerous cell. By increasing $k$, the dose level increases exponentially. Also, by increasing the reliability level, the dose level increases.

\section{Acknowledgment}

The work of the first author is partially supported by the National Science Foundation, DMS1208273. We would like to thank the reviewer for very constructive comments that lead to improvement of the paper.

\section{References}

1. Keyhanian K, Mansoori GA, Rahimpour M (2010) Prospects for Cancer Nanotechnology Treatment by Azurin. Dynamic Biochemistry, Process Biotechnology and Molecular Biology 4: 48-66.

2. Mansoori GA (2005) Principles of Nanotechnology: Molecular-Based Study of Condensed Matter in Small Systems. World Scientific Pub Co Inc.

3. Mansoori GA, Mohazzabi P, McCormack P, Jabbari S (2007) Nanotechnology in cancer prevention, detection and treatment: bright future lies ahead. World Review of Science, Technology and Sustainable Development 4: 226-257.

4. Kuttler F Valnet-Rabier MB Angonin R, Ferrand C, Deconinck E et al. (2002) Relationship between expression of genes involved in cell cycle control and apoptosis in diffuse large B cell lymphoma: a preferential survivin-cyclin B link. Leukemia 16: 726-735.

5. Kanwar JR, Shen WP, Kanwar RK, Berg RW, Krissansen GW (2001) Effects of survivin antagonists on growth of established tumors and B7-1 immunogene therapy. J Natl Cancer Inst 93: 1541-1552.

6. Woynarowska BA, Woynarowski JM (2002) Preferential targeting of apoptosis in tumor versus normal cells. Biochim Biophys Acta 1587: 309-317.

7. Kaufmann SH, Earnshaw WC (2000) Induction of apoptosis by cancer chemotherapy. Exp Cell Res 256: 42-49.

8. Summers HD, Rees P, Holton MD, Brown MR, Chappell SC, et al. (2011) Statistical analysis of nanoparticle dosing in a dynamic cellular system. Nat Nanotechnol 6: 170-174.

9. Hashemian AR, Mansoori GA (2013) Cancer Nanodiagnostics and Nanotherapeutics through the Folate- Conjugated Nanoparticles. J Bioanal
Biomed 5: 061-064

10. Mansoori GA, Brandenberg KS, Shakeri-Zadeh A (2010) A comparative study of two folate-conjugated gold nanoparticles for cancer nanotechnology applications. Cancers (Basel) 2: 1911-1928.

11. Casella G, Berger RL (2002) Statistical inference second edition. Duxbury advanced series.

12. Zink D, Fischer AH, Nickerson JA (2004) Nuclear structure in normal and cancer cells. Nat Rev Cancer 4: 677-687.

13. Letfullin RR, George TF (2013) Plasmonic nanomaterials in nanomedicine. Springer Handbook of nanomaterials :1063-1097.

14. Zharov VP, Lutfullin RR, Galitovskaya EN (2005) Microbubbles-overlapping mode for laser killing of cancer cells with absorbing nanoparticle clusters. J Phys D: Appl Phys 38: 2571-2581.

15. Lawless JF (2002) Statistical models and methods for lifetime data, Second edition. Wiley.

16. R-Manual (2012) The R Project for Statistical Computing 
Appendix 1

It is clear that

$P(W=1)=\int_{0}^{1} p \times P\left(\frac{X}{X+Y}=p\right) d p=E\left(\frac{X}{X+Y}\right) \approx \frac{\lambda_{2}}{\left(\lambda_{1}+\lambda_{2}\right.}$.

Appendix 2

The probability mass function of $L_{1}$ can be written as

$P\left(L_{1}=l\right)=\sum_{l=i}^{c} P\left(L_{1}=l \mid L=i\right) \times P\left(L_{1}=i\right)=\sum_{l=i}^{c} \frac{i !}{(i !)(i-l) !}\left[\frac{\lambda_{2}}{\left(\lambda_{1}+\lambda_{2}\right)}\right]^{l}\left[1-\frac{\lambda_{2}}{\left(\lambda_{1}+\lambda_{2}\right)}\right]^{l-i} \times \frac{c !}{(i !)(c-i) !}\left[p^{*}\right]^{i}\left[1-p^{*}\right]^{c-i}$

$=b(l, c)$,

where $b(l, C)$ is given by the equation

Appendix 3

R codes for calculating $C$.

$\mathrm{z}<-\operatorname{seq}(1, \mathrm{~m}, \mathrm{by}=1) \mathrm{s}$

$\operatorname{sum}\left(1-\operatorname{pnorm}\left(\mathrm{k}^{*} \mathrm{z}+0.5, \mathrm{~A}^{*},\left(A^{*}\right)^{*} 0.5\right)\right) * \frac{\exp (-\lambda p)(\lambda p)^{2}}{z !}$

Note that $\mathrm{m}$ is the smallest integer such that $P\left(Z_{1} \geq m\right)=0$ and $\mathrm{A}^{*}$ is given by the equation (3.2). Also, for large $\lambda p$, to run the program

sometimes we need to approximate $\frac{\exp (-\lambda p)(\lambda p)^{z}}{z !} \operatorname{by} \Phi\left(\frac{z+0.5-\lambda p}{(\lambda p)^{0.5}}\right)-\Phi \frac{z-0.5-\lambda p}{(\lambda p)^{0.5}}$. 